\title{
Winkelsteife Implantate an der HWS
}

\author{
Bernd Vock, Stefan Matschke, Paul Alfred Grützner, Andreas Wentzensen
}

\section{Zusammenfassung}

Die ventrale Spondylodese mit einem winkelsteifen Implantat und monokortikaler Schraubenverankerung nach Interposition eines Spongiosablockes stellt das am häufigsten angewandte Stabilisierungsverfahren bei relevanten Verletzungen der Halswirbelsäule dar. Es resultiert in der Regel eine stabile knöcherne Ausheilung mit gutem funktionellen Ergebnis. Der operative Eingriff hat sich bewährt und ist als Standardverfahren im Bereich der unteren Halswirbelsäule etabliert. Ausgeprägte hintere Instabilitä- ten müssen aber erkannt und der Eingriff mit einer hinteren Stabilisierung verbunden werden. Eine Besonderheit stellen Frakturen bei Morbus Bechterew dar. Bei diesen ist immer eine kombinierte Fusion angezeigt. Bei neurologischen Ausfällen ist der Eingriff dringlich. Mit dem motorbetriebenen isozentischen C-Bogen Siremobil Iso-C-3D ist es möglich, noch intraoperativ das operative Ergebnis bezüglich Implantat-und Knochenblocklage als auch den Spinalkanal in frei wählbaren Bildebenen zu kontrollieren.

weist bei gleichem biomechanischen Verhalten einen einheitlichen Bauplan auf. Unfallfolgen in diesem Abschnitt weisen deshalb Verletzungsmuster mit ähnlichen pathologisch anatomischen Veränderungen auf. Für die gesamte untere Halswirbelsäule hat sich deshalb ein im Wesentlichen einheitliches Therapieverfahren entwickelt.

Stabile Frakturen werden konservativ behandelt. Bei den Frakturen der unteren Halswirbelsäule handelt es sich aber häufiger als in anderen Wirbelsäulenabschnitten bei Beteiligung der Bandscheiben und der Längsbänder um instabile Zustände. Bedingt durch einen engeren Spinalkanal sind neurologische Ausfälle häufig.

alleine oder in Kombination mit einer winkelstabilen Plattenverbindung zwischen C2 und C3 behandelt werden (Abb.1).

Die untere Halswirbelsäule von C3 bis zum Übergang zur Brustwirbelsäule

OP-JOURNAL 2004; 20: 38-44

(c) Georg Thieme Verlag KG Stuttgart · New York

darstellbaren Läsion der anatomischen Strukturen, dem Nachweis einer abnormen Instabilität oder einer dem Halswirbelsäulenbereich zuzuordnenden neurologische Schädigung, die als relevante
Verletzung bezeichnet wird, ist in der Regel die Indikation für ein operatives Vorgehen gegeben. Wenn Veränderungen vorliegen, bei denen durch die operative Stabilisierung eine Besserung eines bestehenden neurologischen Schadens möglich erscheint, ist der Eingriff dringlich [5].

Ligamentäre Schäden heilen meist nicht stabil aus. Deshalb besteht die operative Behandlung in der Regel in einer knöcherne Fusion des oder der betroffenen Bewegungssegmente. Möglich sind ventrale und dorsale Eingriffe oder deren Kombination.

Die ventrale interkorporelle Spondylodese mit Beckenkammblockinterposition und Stabilisierung mittels Platte gilt bei instabilen Verletzungen dieses Abschnittes als Standardverfahren. Ziel ist jeweils die Fusion mindestens eines Bewegungssegmentes (Abb.2).

\section{Geschichtliches}

1891 wurde erstmals durch Hadra die Stabilisierung an der HWS durch Silberdraht propagiert. Orozco stellte Anfang der 70er-Jahre das erste von vorne anzuwendende Plattensystem in H-Form vor. 1986 entwickelte Morscher eine H-Platte mit winkelstabiler Schrauben-Plattenverbindung als Vorläufer der heute von uns benutzten HWS-Verriegelungsplatte mit variablem Winkel von der Firma Synthes ${ }^{\circledR}$.

Die Fusion mittels Knochenblock hat nach den ersten Beschreibungen bei degenerativen Veränderungen von SmithRobinson und Cloward früh Eingang auch bei traumatischen Veränderungen gefunden. Das Verfahren führte aber bei instabilen Verhältnissen ohne zusätzliche Plattenstabilisierung von vorne zu unbefriedigenden Ergebnissen.

Frakturen der unteren HWS sind häufig instabil und werden in der Regel operativ behandelt.

Bei einer durch bildgebende Verfahren

Früh wurde deshalb eine Sicherung des Wirbelsäulensegmentes durch Platten 


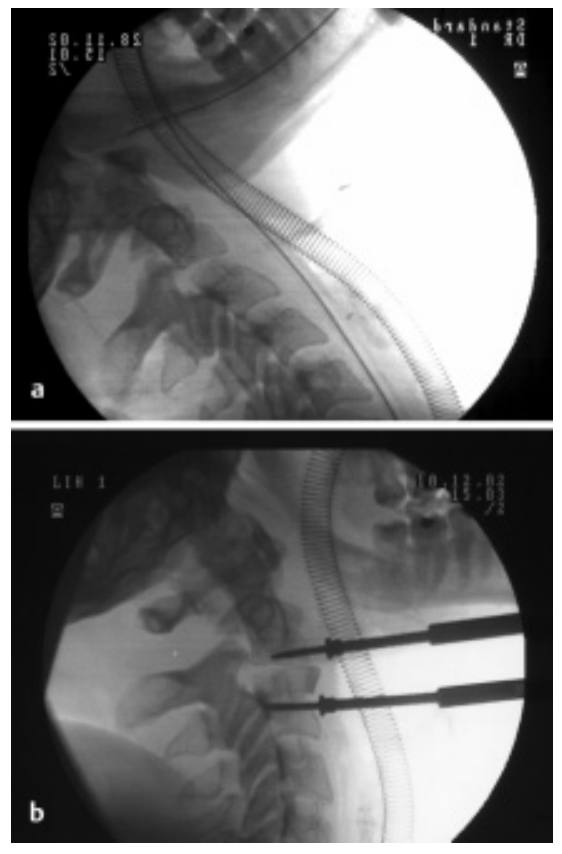

empfohlen. Ein weichteilschonender Zugang und die gute Möglichkeit Bandscheiben- und Knochenfragmente, die in den Spinalkanal eingedrungen waren, unter Sicht zu entfernen sprachen für das Vorgehen von ventral.

Bei unzureichender Stabilität innerhalb des Implantates wurden zur Verbesserung die Schrauben auch in der Hinterkante der Wirbelkörper im Sinne einer bikortikalen Verankerung platziert.

Schrauben- und Plattenlockerungen mit Korrekturverlust und Schäden der umgebenden Weichteile waren dennoch die Folge. Die Schäden betrafen insbesondere die Speiseröhre und reichten bis zur Verlagerung der Implantate in den Gastrointestinaltrakt $[9,12,14]$.

Blauth [2] stellte 1996 Spätergebnisse nach ventralen Fusionen der Jahre 1972 bis 1983 vor. Die Eingriffe waren sowohl ohne Implantat als auch mit nicht winkelstabilen Platten durchgeführt worden. Trotz ungünstiger Ausgangsbedingungen und 6,9\% Komplikationen von Seiten des Implantates waren die Ergebnisse positiv. Möglicherweise war an der hohen knöchernen Ausheilungsrate eine jeweils sehr lange Ruhigstellung, teilweise auch im Minervagipsverband beteiligt.

Im Zeitraum von 1984 bis 1991 wurden in der eigenen Klinik instabile Verletzungen der unteren HWS anfänglich mit dem Orozco-Instrumentarium, später dann mit der von Morscher entwickelten win-

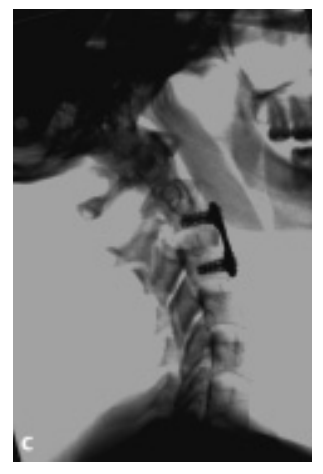

Abb.1 (a) Hangmen's fracture mit Beteiligung des Bandscheibenraumes C2/3. (b) Eingesetzter Beckenkammblock C2/3 mit Distraktor. (c) Ventrale Verriegelungsplatte (d) Gutes funktionelles Ergebnis.
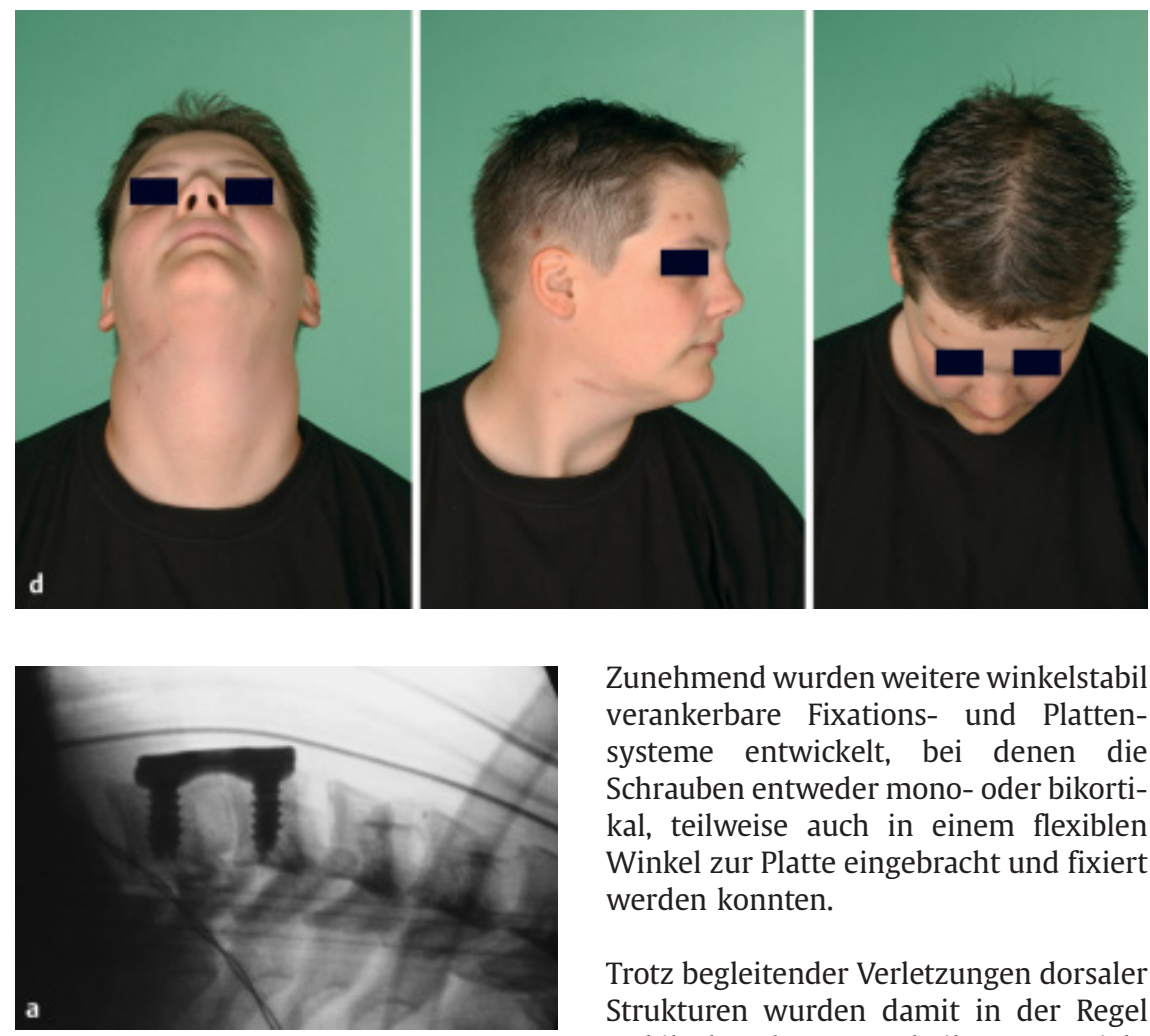

Zunehmend wurden weitere winkelstabil verankerbare Fixations- und Plattensysteme entwickelt, bei denen die Schrauben entweder mono- oder bikortikal, teilweise auch in einem flexiblen Winkel zur Platte eingebracht und fixiert werden konnten.

Trotz begleitender Verletzungen dorsaler Strukturen wurden damit in der Regel stabile knöcherne Ausheilungen erzielt.

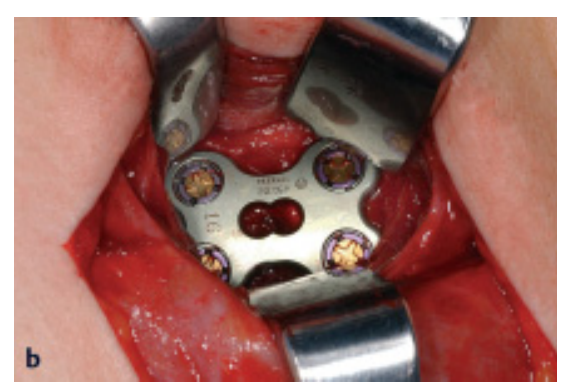

Abb.2au.b Stabilisierung C6/7 mittels HWS-Verriegelungsplatte nach Interposition eines Beckenkammblock.

kelstabilen Platte operativ versorgt. Die Nachuntersuchung von 49 Patienten ergab deutliche Hinweise auf eine Überlegenheit der zuletzt verwendeten winkelstabilen Platte bezüglich Stabilität und knöcherner Heilungstendenz.
Die funktionellen Ergebnisse waren bei begrenzten Bewegungseinschränkungen günstig [7].

Fuchs et al. fanden nach Verwendung eines ventralen winkelstabilen Druckplattenfixateurs bei 131 Patienten in der Regel einen knöchernen Durchbau. Lediglich nach kurzstreckiger Fusion bei Morbus Bechterew und osteoporotischen Veränderungen kam es zu einem Implantatversagen mit Ausriss und Stellungsverlust [10]. Mit einem kleiner dimensionierten Titanfixateur interne $\left(\right.$ Tifix $^{\circledR}$, Litos Hamburg) mit variabel wählbarer Schraubenrichtung hatten sie gleich gute Ergebnisse [11].

Lediglich nach konservativen Versuchen sahen Böhm et al. Fehlschläge mit der Notwendigkeit spätrekonstruktiver Eingriffe [4]. 
Daentzer und Böker [1] fanden nach ventraler Fusion mit dem winkelstabilen Codman ${ }^{\circledR}$ Anterior Cervical Plate System bei allen nachuntersuchten Patienten eine komplette knöcherne Ausheilung.

Ob bei der Verwendung winkelstabiler Implantate eine bikortikale Schraubenfixation eine weitere Zunahme der Steifigkeit bedeutet, wurde kontrovers diskutiert. Blauth wies 1997 bei biomechanischen Untersuchungen eine steifere Montage durch bikortikale Instrumentierung bei einem winkelstabilen Implantat nach [3]. Dagegen fand Rehbein [16] keine weitere Erhöhung der Steifigkeit durch eine bikortikale Verriegelung von winkelsteifen Implantaten.

\section{Behandlungsstrategie}

Bei relevanten Verletzungen der Halswirbelsäule wurden im Rahmen einer Sammelstudie der DGU über einen Zeitraum von $2 \frac{1}{2}$ Jahren Daten zum Zeitablauf der Therapie, zur angewandten Operationstechnik, zu aufgetretenen Komplikationen und insbesondere zu neurologischen Ausfällen und deren Besserungstendenz in Abhängigkeit von Versorgungsmodalitäten erfasst. Die Studie spiegelt die derzeitige gängige Behandlungsstrategie bei Verletzungen der Halswirbelsäule wider [13].

\section{Diagnostik}

Eine eingehende neurologische Untersuchung - insbesondere bei Ausfällen stellt einen wesentlichen Teil der klinischen Untersuchung dar. Neurologische Ausfälle werden entsprechend der ASIA-Klassifikation dokumentiert (Tab.1).
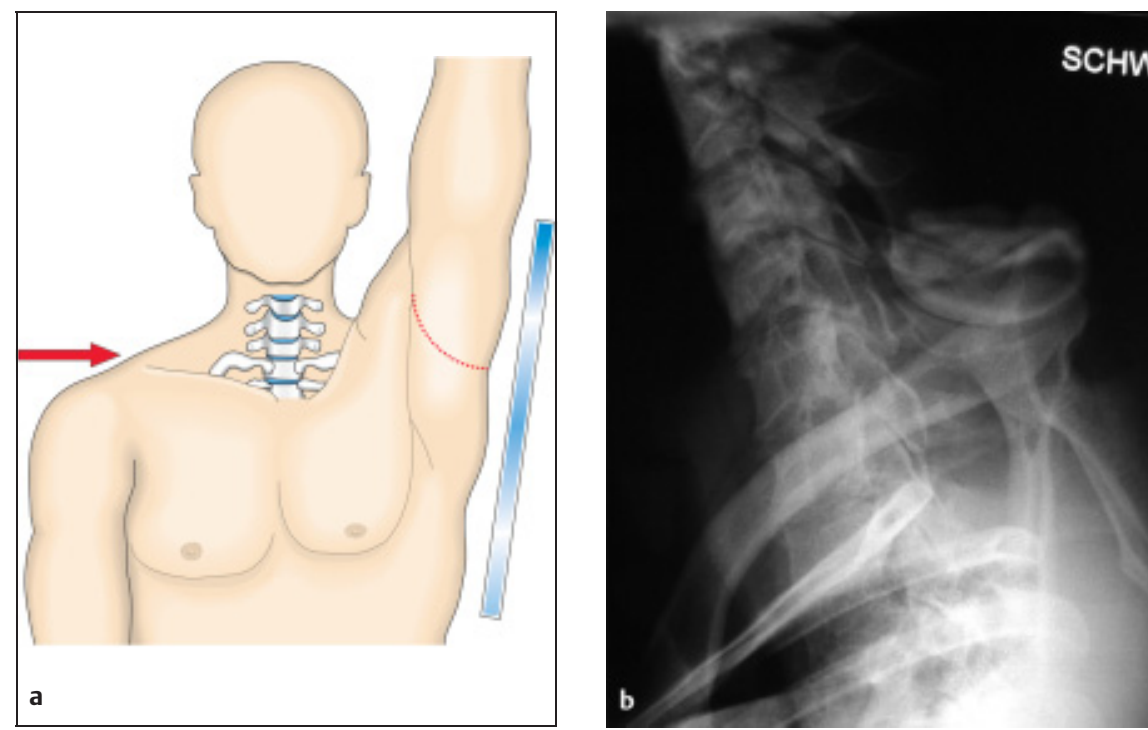

Abb. 3 Röntgenologische Darstellung des zervikothorakalen Überganges in der sog. Schwimmerstellung (a aus OP-Journal 2001; 17: 128-130).
Grundlage der bildgebenden Diagnostik sind Aufnahmen der HWS in 2 Ebenen [15].

Bei der Beurteilung der Röntgenaufnahmen ist auf eine Unterbrechung der zervikalen Begrenzungslinien, auf vermehrte Abstände der Dornfortsatzreihen, auf Knickbildungen und insbesondere auf Frakturen und erkennbare Instabilitäten zu achten. Veränderungen müssen eine weitere abklärende Schnittbilduntersuchung zur Folge haben.

Relevante Verletzungen am Übergang zur Brustwirbelsäule können anfänglich übersehen werden. Ursache ist die problematische Darstellung der unteren HWS im seitlichen Röntgenbild. Durch Zug an den Armen während der Aufnahme oder durch eine so genannte Schwimmerposition kann die Darstellung verbessert werden (Abb.3).

\section{Tab. 1 Die „ASIA Neurological Impairment Scale“}

A Komplett. Keine sensible oder motorische Funktion in den sakralen Segmenten S4 und S5 erhalten.

B Inkomplett. sensible Funktion unterhalb des neurologischen Niveaus und bis in die sakralen Segmente S4 und S5 erhalten.

C Inkomplett. Motorische Funktion unterhalb des neurologischen Niveaus erhalten; die Mehrzahl der Kennmuskeln unterhalb des neurologischen Niveaus haben einen Kraftgrad von weniger als 3.

D Inkomplett. Motorische Funktion unterhalb des neurologischen Niveaus erhalten; die Mehrzahl der Kennmuskeln unterhalb des neurologischen Niveaus haben einen Kraftgrad von gleich oder größer als 3.

E Normal. Sensible und motorische Funktion normal.

ASIA Classification (1992) Standards for neurological and functional classification of spinal cord injury. American Spinal Injuries Association, Chicago, Illinois
Bei unzureichender Darstellung der unteren HWS sind ergänzende Röntgenund Schnittbilduntersuchungen notwendig.

Eine weitere notfallmäßige Abklärung durch Schnittbilduntersuchungen (CT und MRT) ist insbesondere bei Vorliegen eines Nervenschadens unerlässlich. Sagittale Rekonstruktionen zeigen dann häufig die Ursache.

Eine seitliche Durchleuchtung unter Führung des Kopfes durch den Untersucher bereits in der Notaufnahme deckt häufig bereits in der Frühphase eine Instabilität auf. Neben der Höhenbestimmung lässt sich das Ausmaß einer Instabilität besser als in Funktionsuntersuchungen mit seitlichen Röntgenaufnahmen beurteilen [6].

Eine Magnetresonanztomographie lässt in der Akutphase den Schadensort und die Ursache für ein bestehendes neurologisches Defizit eingrenzen, wenn sowohl die Nativröntgendiagnostik als auch eine Computertomographie als Schnittbilduntersuchung den Nervenschaden durch eine feststellbare Fraktur oder Instabilität nicht hinreichend erklären können. Sowohl Bandscheibenschäden als auch intraspinale Hämatome können zur Darstellung kommen.

\section{Wahl des Implantates und der Methode}

Bei der Auswahl des Osteosynthesematerials kommen heute fast ausschließlich winkelstabile Implantate in den verschiedensten Ausführungen infrage. 


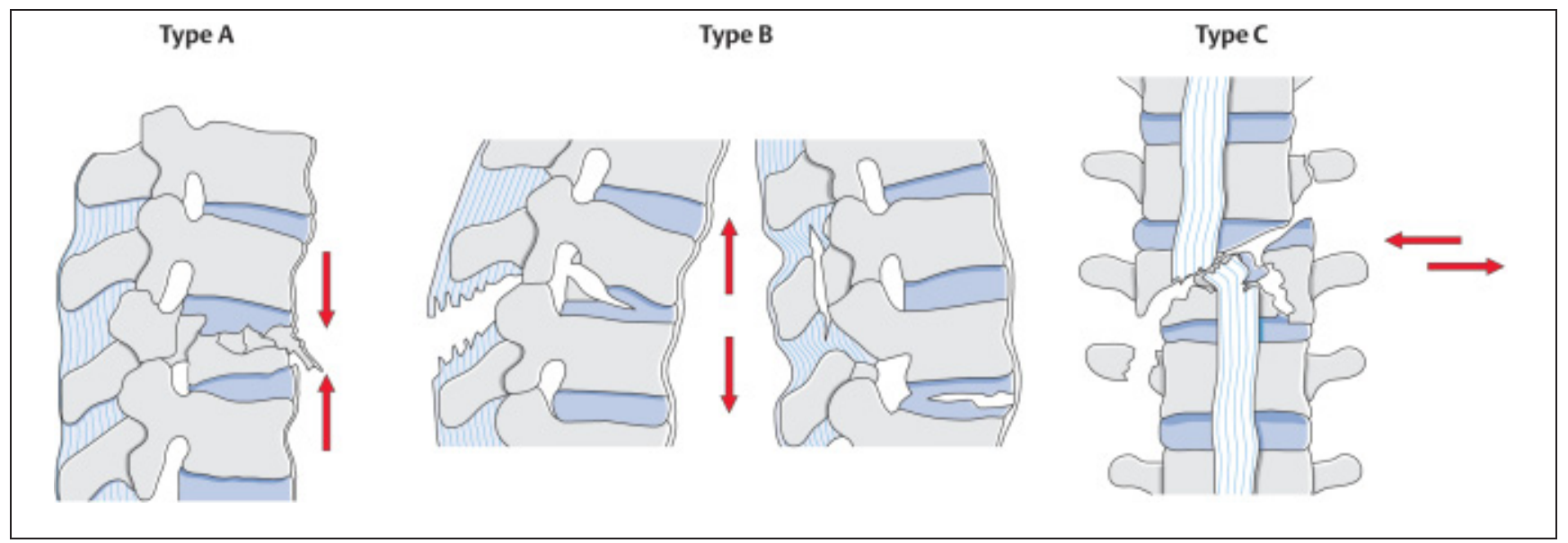

Abb.4 Nach der AO-Einteilung werden an der Halswirbelsäule folgende Verletzungstypen unterschieden: (Type A) Kompressionsverletzung der vorderen Säule. (Type B) Zweisäulenverletzung mit Distraktion. (Type C) Zweisäulenverletzung mit Rotation (OP-JOURNAL 2001; 17: 145).

Das Ausmaß der Überbrückung ist vom Verletzungsmuster abhängig. Bei einer isolierten Instabilität durch Bandruptur und Bandscheibenschädigung reicht eine Fusion der beiden benachbarten Wirbelkörper nach Beckenkammspaninterposition aus. Eine teilweise oder komplette Entfernung und der Ersatz durch einen zurechtgeformten Knochenblock ist bei stärkerer Beschädigung eines Wirbelkörper notwendig. Es resultiert dann eine zweisegmentale Fusion oder eine mehrsegmentale, wenn ausnahmsweise mehr als ein Wirbelkörper frakturiert ist.

\section{Klassifikations-Entscheidungsfindung}

Bei der Entscheidung für ein operatives oder konservatives Vorgehen ist wesentlich, ob die Verletzung stabil oder instabil ist. Hilfreich ist hier die AO-Klassifikation nach Magerl (Abb.4).

Nach der Sammelstudie der Arbeitsgemeinschaft Wirbelsäule wurden A1-Frakturen der unteren Halswirbelsäule überwiegend konservativ und B1-Frakturen gleichverteilt operativ und konservativ behandelt. Bei allen anderen Frakturtypen stand die operative Stabilisierung im Vordergrund. Die Schädigungshöhe C5/6 und C6/7 war am häufigsten betroffen.

Neurologische Ausfälle fanden sich vor allem im Bereich der unteren HWS und korrelierten mit der Verletzungsschwere. Mehr als die Hälfte der C-Verletzungen wiesen inkomplette oder komplette Lähmungen auf. In der Regel wurde der Eingriff als dringlich eingestuft. Bei den klinisch kompletten Lähmungen kam es in $10 \%$ der Fälle postoperativ zu einer Besse- rung um mindestens eine ASIA-Stufe. Bei inkompletten Lähmungen der Stufe $C$ und $\mathrm{D}$ fand sich eine deutliche Besserung bei nahezu der Hälfte der Patienten [13].

Die instabile Bogen-Fraktur des zur oberen HWS gehörenden HWK 2 mit begleitender Bandscheiben- und Längsbandschädigung kann in gleicher Weise stabilisiert werden.

Die Indikation zur operativen Stabilisierung ergab sich einerseits aus der Verletzungsschwere entsprechend der Klassifikation nach Magerl, andererseits aus einer klinisch bestehenden Instabilität.

In der genannten Studie war die vordere Versteifungsoperation (Spondylodese) mit Beckenkammblock und Plattenstabilisierung das Standardverfahren. Meist handelte es sich um die Versteifung eines betroffenen Bewegungssegmentes (monosegmentale Fusion) nach Ausräumung der Bandscheibe. Bei stärkerer Beschädigung eines Wirbelkörpers wurde dieser teilentfernt, durch einen entsprechend größeren Knochenblock ersetzt und eine mehrsegmentale Fusion durchgeführt.

Meist ist eine monosegmentale Fusion von vorne ausreichend.

Liegt eine Schädigung des Halsrückenmarkes vor, sollte der letzte intakt innervierte Rumpfabschnitt durch einen dorsalen Zugang nicht zusätzlich geschädigt werden. Auf zusätzliche dorsale Stabilisierungsverfahren wurde lediglich bei hochgradigen hinteren Instabilitäten, bei von vorne nicht reponiblen Verrenkungen, bei alten verhakten dorsalen Lu- xationen oder bei Verletzungen, die sich erst im Verlauf als hoch gradig instabil erwiesen, zurückgegriffen. Eine Besonderheit stellen Frakturen bei M. Bechterew dar. Diese müssen immer als hoch gradig instabil eingeschätzt werden. Sowohl die vordere als auch die hintere Stabilisierung muss dann langstreckig erfolgen. Die hintere Stabilisierung kann mit Haken-, Platten- oder Stabsystemen durchgeführt werden (Abb.5).
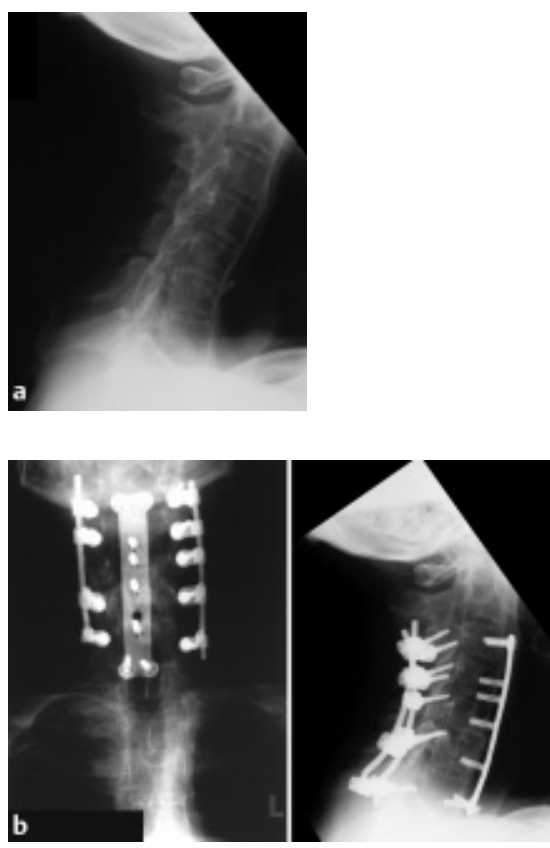

Abb. 5 (a) Hochgradige Instabilität C5/6 bei Morbus Bechterew. (b) Zusätzliche dorsale Fusion mittels Stabsystem. Beginnende Schraubenlockerung $\mathrm{C} 7$. 


\section{Operativer Ablauf}

Im eigenen Krankengut wird seit 14 Jahren die HWS-Verriegelungsplatte der Fa. Synthes ${ }^{\circledR}$ verwendet. Spreizkonusse sichern in monokortikal eingebrachten Spreizkopfschrauben die Winkelstabilität. Die Platte wurde im Verlaufe weiterentwickelt. Jetzt ermöglicht ein Schwenkring im Plattenloch das Setzen der Schrauben in einem bis $20^{\circ}$ variablen Winkel. Bikortikale HWS-Spreizkopfschrauben sind bei ausgeprägten Instabilitäten verfügbar.

Adaptiert an das Verletzungsmuster wird die Fusion ein- oder mehrsegmental durchgeführt. Bei den mehrsegmentalen Fusionen sollte bei nicht ausreichender Dekompression die Hinterwand entfernt werden.

Verletzungen bei Morbus Bechterew gelten als hoch gradig instabil. Sie erfordern immer eine kombinierte langstreckige Fusion [8]. Auch Verletzungen, die mit einer ausgeprägten dorsalen Instabilität vergesellschaftet sind, müssen kombiniert dorsoventral stabilisiert werden.

- Der Patient wird nach Unterpolsterung der Schulterblätter auf dem Rücken gelagert. Für eine ungehinderte Durchleuchtung des Überganges zur BWS erfolgt an den Armen ein Dauerzug.

- Die Halswirbelsäule wird für eine längerstreckige Fusion nach Längsinszision vor dem M. sternocleidomastoideus zwischen den medial gelegenen viszeralen Strukturen und dem lateralen Gefäß-Nerven-Bündel erreicht. Für eine kurzstreckige Fusion reicht eine mehr quer verlaufende Inzision in Höhe der Läsion aus.

- Nach Darstellung der HWS wird ein Distraktor in die benachbarten Wirbel eingesetzt. Bei monosegmentaler Fusion wird danach der Bandscheibenraum ausgeräumt.

- Ein kortikospongiöser Knochenblock wird am Beckenkamm entnommen zurechtgeformt und in den Bandscheibenraum eingesetzt ohne ihn dabei zu distrahieren.

- Nach Bestimmung der korrekten Implantatlänge wird die HWS-Verriegelungsplatte mit selbstbohrenden und -schneidenden Schrauben monokortikal und winkelstabil besetzt.

- Ein zusätzlicher hinterer Zugang mit Fusion ist bei nicht reponiblen dorsalen Verhakungen oder bei höher gradigen Instabilitäten nach Umlagerung notwendig.

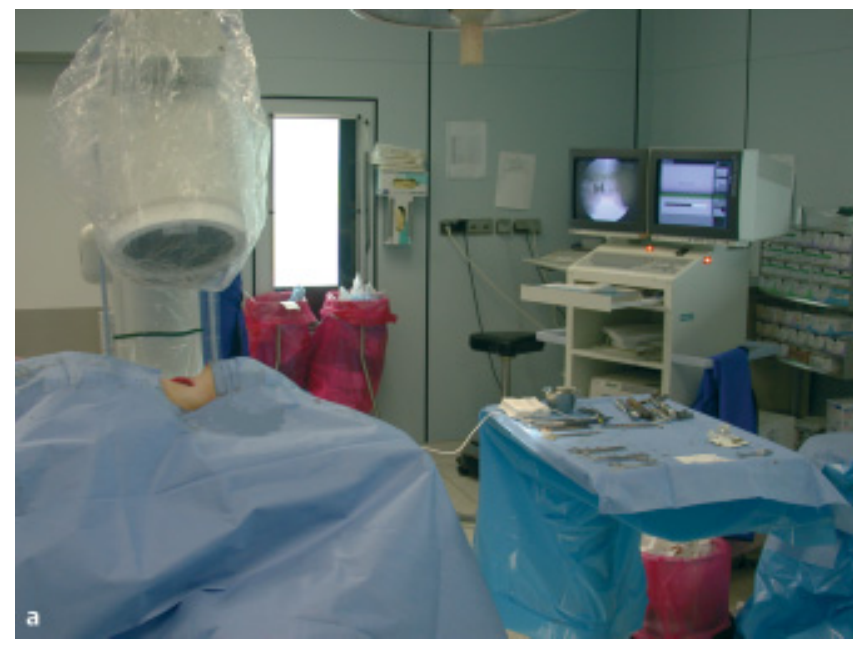

Abb. 6 (a) Intraoperative 3D-Darstellung des Spongiosablockes, des Implantates und des Spinalkanales mit dem Siremobil Iso-C3D. (b) Regelrechte Verhältnisse in axialer, frontaler Ansicht.
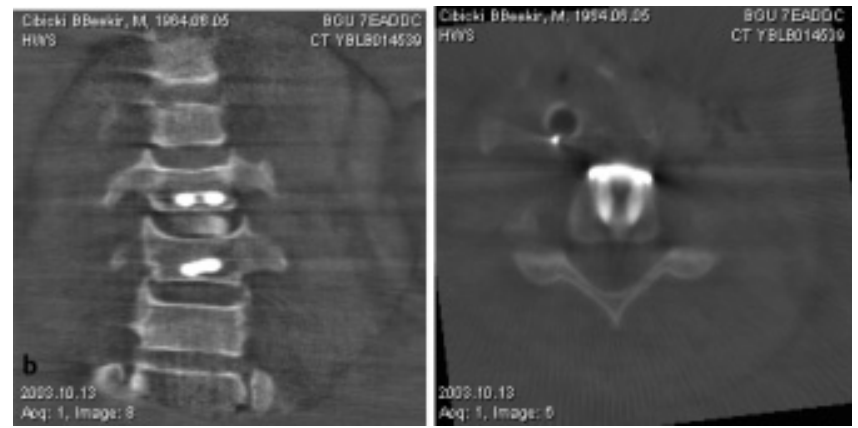

Vor dem Wundverschluss erfolgt nach Fertigstellung der Montage eine Kontrolle in konventionellen zweidimensionalen Durchleuchtungsbildern. Eine postoperative Computertomographie lässt eine differenzierte Beurteilung des Operationsergebnisses $\mathrm{zu}$.

In der eigenen Klinik steht mit dem 3D-CBogen Siremobil Iso-C-3D der Firma Siemens ein Gerät zur Verfügung, das neben der konventionellen 2D-Durchleuchtung die intraoperative 3D-Darstellung knöcherner Strukturen erlaubt. Es handelt sich um einen mobilen C-Bogen mit Isozentrizität und $190^{\circ}$ Orbitalbewegung. Im 3D-Betrieb werden während einer motorgesteuerten kontinuierlichen orbitalen Rotation um $190^{\circ}$ eine definierte Zahl von Durchleuchtungsbildern in festen Winkelabständen aufgenommen. Mit den 2D-Durchleuchtungsdaten wird ein hochaufgelöster 3D-Datenwürfel im Isozentrum des C-Bogens errechnet. Die Kantenlänge des generierten Würfels beträgt $12 \mathrm{~cm}$. In diesem Ausmaß lassen sich noch intraoperativ beliebige Schnittebenen in Echtzeit darstellen. Noch vor dem Weichteilverschluss ist eine Kontrolle der Lage des Implantates, des Knochenblockes als auch eine Beurteilung des Spinalkanals in frei wählbaren Ebenen mög- lich. Gegenüber einer konventionellen Durchleuchtung ist der zeitliche Mehraufwand mit ungefähr 2 Minuten begrenzt. In der Regel ist danach eine postoperative CT-Untersuchung entbehrlich (Abb. 6).

Eine intraoperative 3-D-Darstellung ergibt eine zusätzliche Sicherheit vor dem Weichteilverschluss.

\section{Eigene Erfahrungen}

Bei 82 Unfallverletzten wurde im Zeitraum von 1995 bis 2002 aufgrund einer Instabilität im Bereich der unteren HWS eine ventrale Spondylodese durchgeführt.

Überwiegend handelte es sich um Fusionen des Segmentes C5/6. Zwei- und mehrsegmentale Eingriffe waren die Ausnahme. In drei Fällen war ein kombinierter Eingriff wegen einer höher gradigen Instabilität notwendig. Das alleinige ventrale Vorgehen mit winkelsteiferer Platte und Spongiosablock war in allen anderen Fällen ausreichend, eine knöcherne Verbindung zu erreichen.

Trotz langstreckiger kombinierter Eingriffe bereiteten gerade Frakturen beim 

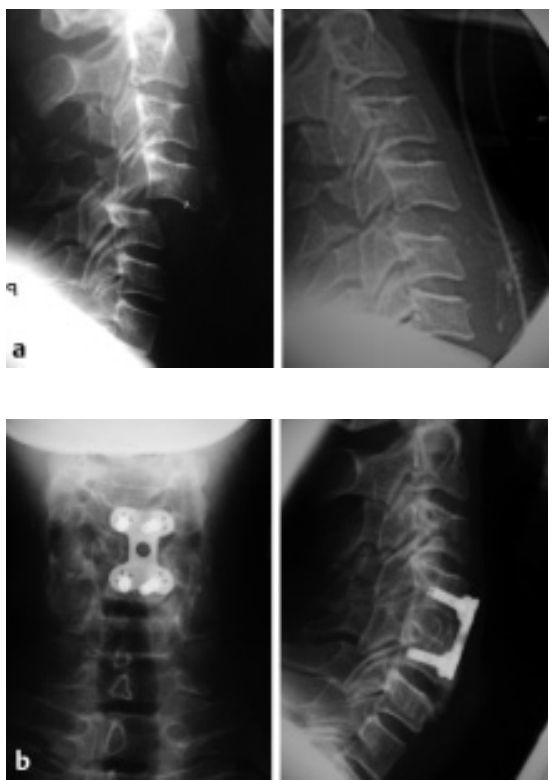

Abb.7 (a) Luxation C4/5 mit kompletter Tetraplegie und geschlossene Reposition innerhalb einer Stunde nach Unfall. (b) Ventrale Spondylodese und gute neurologische Erholung.

Morbus Bechterew Probleme. Frühe Implantatlockerungen konnten beobachtet werden. Ohne weitere operative Maßnahmen waren dennoch knöcherne Heilungen das Ergebnis.

\section{Neurologischer Schaden}

Die mit einem winkelsteifen Implantat von vorne operierten Patienten wiesen mit 55\% der Fälle einen inkompletten oder kompletten Nervenschaden auf. Ursache für den hohen Anteil von Lähmungen dürften die anatomischen Gegebenheiten mit im unteren HWS-Bereich schmaler werdendem Spinalkanal und dem höheren Anteil von instabilen Verletzungen sein.

Verletzte im Zeitraum von 1995 bis Ende 1997 mit neurologischem Defizit wurden bezüglich einer neurologischen Erholung nachuntersucht. Bei 14 von 15 Patienten nach Schädigung im Bereich der unteren HWS wurde eine ventrale Spondylodese mit winkelsteifer Platte durchgeführt. 4 der Patienten wiesen initial einen motorisch kompletten, sensibel einen inkompletten Schaden auf. Bei 10 Patienten bestand anfangs ein komplettes sensomotorisches Querschnittsyndrom. Im Verlaufe zeigte sich bei $2 / 3$ der Patienten eine neurologische Erholung zwischen vier Stufen (1 Patient) und einer Stufe (4 Patienten) (ASIA Impairment Scale). Das günstigste Ergebnis wurde nach einer
C4/5-Luxation erzielt, die sofort nach Eintreffen im Schockraum noch innerhalb einer Stunde nach dem Unfallereignis reponiert wurde (Abb. 7).

Bei einer Querschnittssymptomatik ist der operative Eingriff dringlich.

\section{Diskussion}

Für die Mehrzahl der Verletzungen der unteren Halswirbelsäule als auch der instabilen Bogenfraktur des HWK 2 haben sich weit gehend einheitliche Verfahren herausgebildet. Unter Verwendung eines ventralen vorwiegend winkelsteifen und monokortikalen Plattensystemes in Verbindung mit einem Beckenkammblock ist die ventrale Spondylodese etabliert. Nur in Ausnahmefällen muss ein zusätzlicher Eingriff von hinten durchgeführt werden. Die in der Literatur angegebenen guten Ergebnisse bei der ventralen Spondylodese mit winkelsteifer Platte und kortikospongiösem Block werden durch die eigen Erfahrungen mit der HWS-Verriegelungsplatte der Fa. Synthes bestätigt.

Durch die Beschränkung auf das ventrale Verfahren wird eine zusätzlich Schädigung der dorsalen Weichteile vermieden. Gerade bei Patienten mit neurologischen Schäden ist dies wichtig. Wenige Fehlschläge mit Versagen des Implantates wurden im eigenen Krankengut beobachtet. In jedem einzelnen Fall zeigte sich, dass eine hoch gradige Instabilität anfänglich nicht erkannt wurde und $\mathrm{zu}$ einer Überforderung des alleinigen ventralen Verfahrens führte.

Hintere Instabilitäten müssen erkannt werden und bedürfen einer kombinierten Fusion.

Die sofortige Reposition mit Dekompression schien im Einzelfall den neurologischen Verlauf günstig beeinflusst zu haben. Es fehlen aber statistisch gesicherte Untersuchungsergebnisse, die dies belegen. Zweifellos werden aber durch eine frühzeitige operative Intervention mit Stabilisierung Sekundärschäden vermieden und die Rehabilitation erleichtert.

\section{Schlussfolgerung}

Verletzungen der unteren Halswirbelsäule sind häufig instabil. Meist ist eine operative Stabilisierung angezeigt. Der Eingriff ist bei Verletzungen mit neurologischen Schäden dringlich, wenn die sofortige Intervention eine Besserung zumindest erhoffen lässt.
Die alleinige Spondylodese von vorne mit einer winkelsteifen Platte und einem Beckenkammspan ist in fast allen Fällen ausreichend. Eine zusätzliche hintere Stabilisierung ist in der Regel nicht notwendig. Winkelsteife Plattensysteme ergeben in Verbindung mit dem Beckenkammknochenblock hohe Stabilität und große Sicherheit. Es resultiert bei der ventralen Spondylodese eine gute knöcherne Einheilung des autologen Knochens und meist ein gutes funktionelles Ergebnis.

Hoch gradig instabile Verletzungen, bei denen die Stabilisierung von vorne nicht ausreicht, müssen sofort erkannt und dann auch von hinten hinten operativ stabilisiert werden.

Neuere Entwicklungen in der Bildgebung, die eine intraoperative Lagekontrolle des Implantates, des Knochenblockes und eine Beurteilung des Spinalkanales in 3D-Bildern ermöglichen, geben eine zusätzliche Sicherheit.

\section{Literatur}

${ }^{1}$ Daentzer D, Böker DK. Erfahrungen mit einem ventralen winkelinstabilen monokortikalen Schrauben-Platten-System (ACPS) bei 78 Patienten mit traumatischer Instabilität der HWS. European Journal of TraumaE-Suppl 2002; 1: 34-37

2 Blauth M, Schmidt U, Dienst M, Knop C, Lobenhoffer $\mathrm{P}$, Tscherne $\mathrm{H}$. Langzeitergebnisse von 57 Patienten nach ventraler interkorporeller Spondylodese der unteren Halswirbelsäule. Unfallchirurg 1996; 99: 925-939

${ }^{3}$ Blauth M, Knop C, Bastian L, Lobenhoffer P. Neue Entwicklungen in der Chirurgie der verletzten Wirbelsäule. Orthopäde 1997; 26: $437-449$

${ }^{4}$ Böhm HJ, Kortmann HR, Westerhoff CHA. Spätrekonstruktive Eingriffe nach Verletzungen der Halswirbelsäule. Trauma und Berufskrankheit 2000; 2: 157-161

${ }^{5}$ Bühren V, Hofmeister M, Militz M, Potulski M. Indikationsstellung für die operative Behandlung bei Verletzungen der Halswirbelsäule. Zentralbl Chir 1998; 123: 907 -913

${ }^{6}$ Bühren V. Frakturen und Instabilitäten der Halswirbelsäule. Unfallchirurg 2002; 105 1049-1066

Chylarecki C, Kortmann HR, Hiersemann K, Scheele H, Meyer I. Funktionelle Ergebnisse nach ventraler Spondylodese der unteren Halswirbelsäule bei ossären und diskoligamentären Verletzungen. Trauma und Berufskrankheit 2000; 2: 148-156

${ }^{8}$ Einsiedel T, Kleimann M, Nothofer W, Neugebauer $\mathrm{R}$. Besonderheiten der Therapie von Verletzungen der Halswirbelsäule bei Spondylitis ankylosans (M. Bechterew). Unfallchirurg 2001; 104: 1129-1133

${ }^{9}$ Feeser R, Wolter D, Biermann E. Die transorale Metallentfernung nach ventraler Spondylodese C2/C3 bei pharyngovertebraler Fistel. Unfallchirurg 1997; 100: 984-989

10 Fuchs S, Wolter D, Kortmann HR, Faschingbauer $M$, Jürgens $C$. Ventrale Spondylodese der HWS (C2-C7) mit einem Druckplattenfi- 
xateur interne. Trauma und Berufskrankheit 1999; 1: 375-381

${ }^{11}$ Fuchs S, Wolter D, Wenzl M E, Faschingbauer M. Klinische Erfahrung mit einem neuen Titanfixateur interne zur ventralen Spondylodese der HWS. Trauma und Berufskrankheit 1999; 1 : $382-386$

${ }^{12}$ Fujibayashi S, Shikata J, Kamiya N, Tanaka C. Missing anterior cervical plate and screws: case report. Spine 2000; 25: 2258-2261

${ }^{13}$ Hofmeister M, Bühren V. Therapiekonzept für Verletzungen der unteren HWS- Unter Berücksichtigung der Daten einer multizentrischen Sammelstudie der Arbeitsgemeinschaft Wirbelsäulenchirurgie der DGU. Orthopäde 1999; 28: 401 -413
${ }^{14}$ Müller CA, Strohm PC, Pfister U. Ösophagusperforation durch Osteosynthesematerial nach ventraler Spondylodese der HWS. Unfallchirurg 2001; 104: 549-552

15 Oberst M, Wittner B, Holz U. Frakturen und Luxationen der unteren Halswirbelsäule. OP-Journal 2001; 17: 142-149

${ }^{16}$ Rehbein Ph, Nassutt R, Sellenschloh K, Morlock M, Eggers C. Vergleichende biomechanische Untersuchungen zur Primärstabilität von winkelstabilen und nicht winkelstabilen ventralen Rekonstruktionsmethoden für die Halswirbelsäule. Norddeutscher Orthopädenkongress 1999
Dr. med. Bernd Vock

Oberarzt

Dr. med. Stefan Matschke

Oberarzt

Dr. med. Paul Alfred Grützner

Oberarzt

Prof. Dr. med. Andreas Wentzensen

Ärztlicher Direktor

Klinik für Unfall- und Wiederherstellungschirurgie

BG-Unfallklinik Ludwigshafen

Ludwig-Guttmann-Straße 13

D-67071 Ludwigshafen 